\title{
Clear cell myoepithelial carcinoma in the base of the tongue: Case report and review of the literature
}

\author{
JUN HEE PARK $^{1}$, JI YUN CHOI $^{1}$, RAN HONG $^{2}$ and NAM YONG DO ${ }^{1}$ \\ Departments of ${ }^{1}$ Otorhinolaryngology-Head and Neck Surgery and ${ }^{2}$ Pathology, \\ Chosun University College of Medicine, Gwangju, Republic of Korea
}

Received April 25, 2012; Accepted July 26, 2012

DOI: $10.3892 / \mathrm{ol} .2012 .900$

\begin{abstract}
Clear cell myoepithelial carcinoma is a very rare disease which develops primarily in the parotid gland. To date, only 17 cases of clear cell myoepithelial carcinoma have been reported worldwide. Among them, only three cases developed in the minor salivary gland in the oral cavity. No cases developed in the base of the tongue. Here, we report a new case of clear cell myoepithelial carcinoma that developed in the tongue base of a 52-year-old female patient. A mass was discovered on the left side of the tongue base. We successfully removed the mass through suprahyoid pharyngotomy approach. The light microscopy examination and various immunohistochemical stainings revealed clear cell myoepithelial carcinoma. During a two year follow-up period, there was no recurrence or local or distant metastasis.
\end{abstract}

\section{Introduction}

Myoepithelial carcinoma is a very rare disease that comprises only $2 \%$ of all salivary gland carcinomas. In the majority of cases, it occurs in the parotid gland; however, it occasionally occurs in the minor salivary gland (1).

Myoepithelial carcinoma may present with diverse types of tumor cells including eosinophilic, epithelioid, polygonal, spindle, stellate, hyaline and clear cells. Among them, lesions that display clear cell-type tumor cells focally or predominantly are referred to as clear cell myoepithelial carcinomas (CCMC) (2,3). To date, only 17 cases of CCMC have been reported worldwide. Until the present study, no case has involved the tongue base (2-7). We present a case of CMCC that developed in the base of the tongue. Written informed consent for publication was obtained from the patient.

Correspondence to: Professor Jun Hee Park, Department of Otorhinolaryngology-Head and Neck Surgery, Chosun University College of Medicine, 588 Seoseok-dong, Dong-gu, Gwangju 501-717, Republic of Korea

E-mail: entjh28@hanmail.net

Key words: clear cell carcinoma, myoepithelial carcinoma, clear cell, salivary gland, tongue

\section{Case report}

A 52-year-old female visited Chosun University Hospital (Gwangju, Korea) complaining of the sensation of foreign bodies in the pharynx that had developed several months previously. The patient's history and family history were unremarkable. Upon physical examination, a mass $3 \times 3 \mathrm{~cm}$ in size, with an unclear boundary and no associated ulcer, was discovered on the left side of the tongue base (Fig. 1). No other significant findings in the nasal cavity or the neck area were observed. The results of the general blood tests were normal. Computed tomography (CT) revealed necrosis in the central area of the left side of the tongue base and a $2.9 \times 2.6 \mathrm{~cm}$ circular lesion showing contrast enhancement was also detected (Fig. 2). As several colloidal cysts were detected in both thyroids, ultrasonography was performed. The results were clinically insignificant.

A biopsy was performed under local anesthesia at our outpatient clinic due to suspected adenoid cystic carcinoma. To assess systemic metastasis and other associated diseases, ${ }^{18} \mathrm{~F}$-fluorodeoxyglucose positron emission tomography/CT was performed. There was no evidence of metastasis in the neck or systemic metastasis.

Under general anesthesia, the mass was removed through suprahyoid pharyngotomy. Tracheostomy and primary suture were also performed. The patient was discharged 10 days after surgery without any significant complications.

Macroscopically, the removed mass was light yellow in color and $4.5 \times 3 \mathrm{~cm}$ in size. At the time of resection, a $1.5 \times 1.2 \mathrm{~cm}$ zone filled with red fluid was detected (Fig. 3). Under light microscopy, the mass was partitioned by fibrous septa and contained clear cells that formed a pattern of nests, sheets and cords. The clear cells were polygonally shaped and contained abundant, clear cytoplasm. By immunohistochemical staining, the tumor cells were revealed to be positive for cytokeratin 7 (CK 7), p63 and high molecular weight cytokeratin (HMWCK), and revealed focally weak responses to cytokeratin (Fig. 4). Additionally, the tumor cells revealed negative reactions to S-100 and smooth muscle actin (SMA). As a result of the light microscopy examination and immunohistochemical staining results, a diagnosis of CCMC was made.

The patient has been under follow-up observation for 2 years without recurrence or local or distant metastasis. 


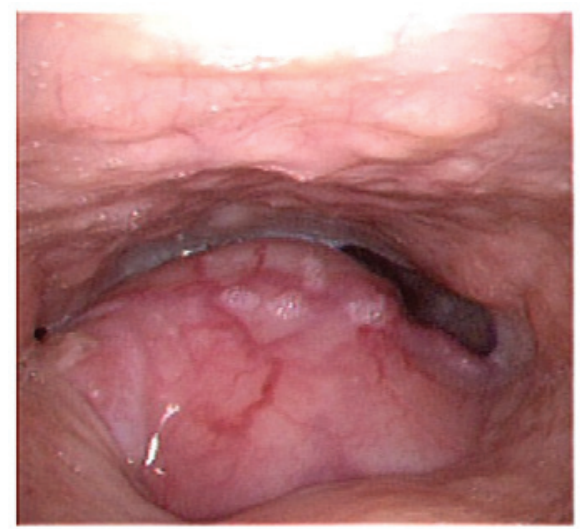

Figure 1. Endoscopic finding of cancer of the tongue base. A protruding mass approximately $3 \times 3 \mathrm{~cm}$ in size is visible on the base of the tongue.
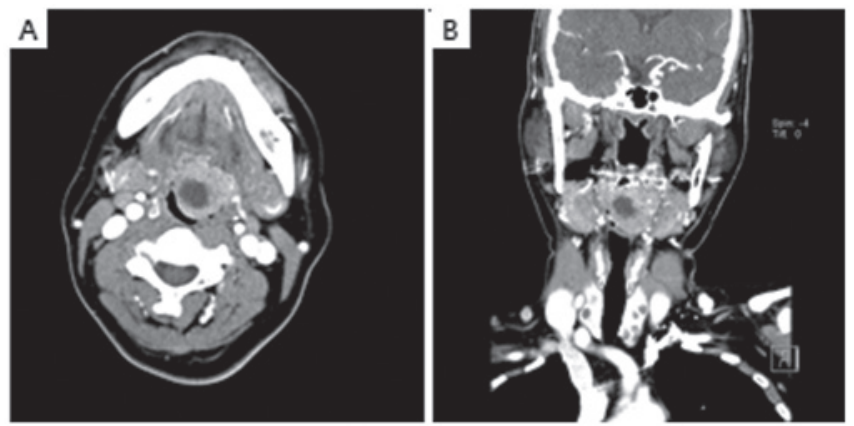

Figure 2. Results of (A) axial and (B) coronal CT scans with contrast enhancement. An enhanced mass approximately $2.9 \times 2.6 \mathrm{~cm}$ in size with internal necrotic low density on the left side of the tongue base is visible.

\section{Discussion}

In salivary gland tumors, myoepithelial differentiation is relatively common. However, cases in which the tumors themselves are primarily formed by myoepithelial cells are very rare. Furthermore, among salivary gland myoepithelial carcinomas, CCMC which presents with almost exclusively the phenotype of clear cells is also extremely rare. Hence, CCMC is a clear cell neoplasm formed by tumor cells with myoepithelial differentiation that presents with a epithelial phenotype and smooth muscle phenotype only $(6,8,9)$. To date, only 17 cases of CCMC have been reported. By examination of the site of tumor development, 9 cases involved the parotid gland, 4 cases involved the submandibular gland and one case involved the maxillary sinus. Three cases occurred in the oral cavity, 2 cases occurred in the palate and one case occurred in the retromolar region (2-7). This is the first study to report a case of CCMC in the base of the tongue.

CCMC displays diverse histological patterns that include nests, cords, trabeculae and sheets. The central area is paucicellular and the peripheral area is hypercellular. Vacuolated clear cells display signet ring or lipoblast shapes $(2,3)$. In the majority of cases, clear tumor cells predominate. However, certain components of myoepithelial carcinoma, spindle cells or nonclear epithelioid cells may appear in certain areas.

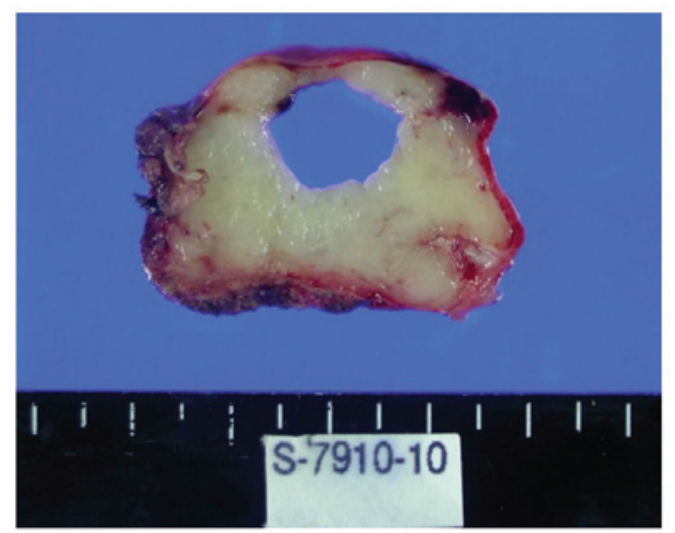

Figure 3. Appearance of the gross specimen. The specimen consists of a portion of the tongue measuring $4.5 \times 3 \times 3 \mathrm{~cm}$ including the mass.

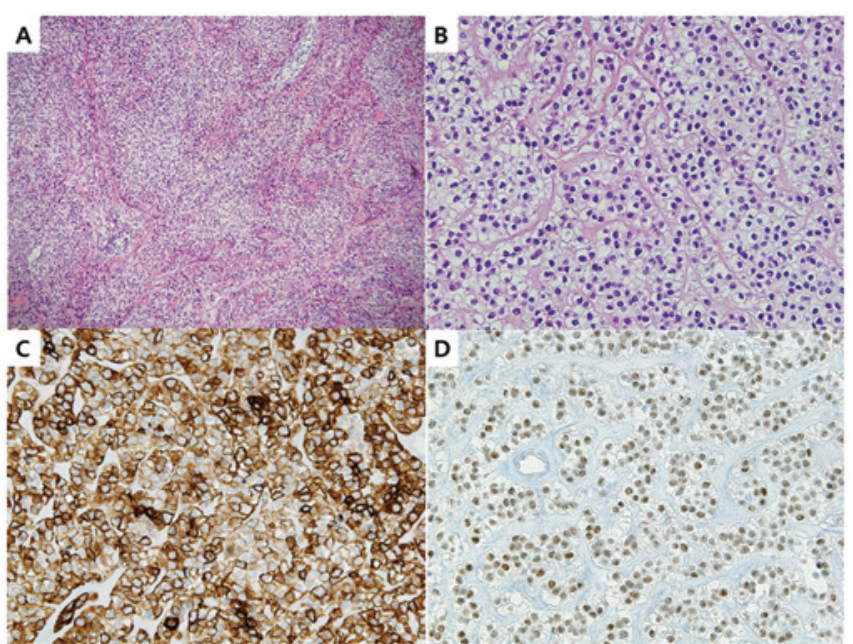

Figure 4. Microscopic appearance of the tumor. The clear cells were polygonally shaped with abundant, clear cytoplasm. (A) x40, H\&E staining; (B) $x 200, H \& E$ staining. Immunohistochemically, the tumor cells show strong membranous staining for CK7 [(C) x200] and nuclear staining for the myoepithelial marker p63 [(D) x200].

Myoepithelial differentiation by immunohistochemical staining is possible as the cells reveal positive reactions to epithelial markers (epithelial membrane antigen and cytokeratin) and at least two of the myoepithelial markers (S-100 protein, calponin, p63, glial fibrillary acidic protein, maspin and actin). Myoepithelial carcinoma reacts most sensitively to vimentin, S-100 and HMWCK $(3,6)$. Furthermore, it has been recently suggested that the p63 and maspin members of the $\mathrm{p} 53$ family of nuclear proteins may be new myoepithelial markers (6). Our case was positive for CK7, p63 and HMWCK and revealed focal weak reactions to $\mathrm{CK}$.

CCMC should be differentiated from other salivary gland tumors that contain clear cell components and from odontogenic and metastatic tumors. Acinic cell carcinoma, high-grade mucoepidermoid carcinoma, sebaceous carcinoma and clear cell oncocytoma that develop in the salivary gland are negative for myoepithelial markers and should therefore readily be capable of being differentially diagnosed. Additionally, 
metastatic clear cell renal cell carcinoma with the clear cell phenotype invading the salivary gland, presents with prominent sinusoidal vascularity and hemorrhage within tumors and is evident under light microscopy examination, which allows for a differential diagnosis $(2,7,10)$.

Based on the treatment for myoepithelial carcinoma, the basic treatment for CCMC is wide surgical excision in the primary tumor site. If metastasis in the cervical lymph nodes is not detected, dissection of the lymph nodes is unnecessary $(3,11)$. Additional postsurgical radiotherapy does not improve the overall prognosis. Nonetheless, it can be administered for the purpose of local control in cases in which an intact safety margin is not secured during surgery, or for cases in which invasion into adjacent tissues is confirmed by microscopic examination. Additionally, postsurgical chemotherapy is administered to treat systemic metastasis, similar to other cancers $(3,11)$. In the present case, lymph node metastasis in other areas, including the neck, was not detected in presurgical tests and a tumor-free margin could be secured by wide surgical excision. Thus, additional postsurgical treatments were not administered.

The prognosis of CCMC remains unclear due to the small number of reported cases. However, a study conducted on myoepithelial carcinoma that reported a $47 \%$ metastatic rate and $29 \%$ mortality rate revealed clinically aggressive patterns (5). Another study that was conducted on CCMC alone reported a $50 \%$ recurrence rate, metastasis in the lung and the scalp and a $40 \%$ metastatic rate (12).

A differential diagnosis of CCMC from other primary tumors containing clear cells and metastatic lesions is essential. When a case is diagnosed as CCMC, aggressive treatments including surgical excision are required.

\section{References}

1. Skalova A and Jakel KT: Myoepithelial carcinoma. In: WHO Classification of Tumours. Pathology and Genetics of Head and Neck Tumours. Barnes L, Eveson JW, Reichart P and Sidransky D (eds). IARC Press, Lyon, pp240-241, 2005.

2. Michal M, Skalova A, Simpson RH, Rychterova V and Leivo I: Clear cell malignant myoepithelioma of the salivary glands. Histopathology 28: 309-315, 1996.

3. Savera AT, Sloman A, Huvos AG and Klimstra DS: Myoepithelial carcinoma of the salivary glands: a clinicopathologic study of 25 patients. Am J Surg Pathol 24: 761-774, 2000.

4. Yang S, Zeng M, Zhang J and Chen X: Clear cell myoepithelial carcinoma of minor salivary gland: a case report. Int $\mathrm{J}$ Oral Maxillofac Surg 39: 297-300, 2010.

5. Losito NS, Botti G, Ionna F, Pasquinelli G, Minenna P and Bisceglia M: Clear-cell myoepithelial carcinoma of the salivary glands: a clinicopathologic, immunohistochemical, and ultrastructural study of two cases involving the submandibular gland with review of the literature. Pathol Res Pract 204: 335-344, 2008.

6. Savera AT and Zarbo RJ: Defining the role of myoepithelium in salivary gland neoplasia. Adv Anat Pathol 11: 69-85, 2004.

7. Wang B, Brandwein M, Gordon R, Robinson R, Urken M and Zarbo RJ: Primary salivary clear cell tumors - a diagnostic approach: a clinicopathologic and immunohistochemical study of 20 patients with clear cell carcinoma, clear cell myoepithelial carcinoma, and epithelial-myoepithelial carcinoma. Arch Pathol Lab Med 126: 676-685, 2002.

8. Dardick I, Thomas MJ and van Nostrand AW: Myoepithelioma new concepts of histology and classification: a light and electron microscopic study. Ultrastruct Pathol 13: 187-224, 1989.

9. Dardick I: Myoepithelioma: definitions and diagnostic criteria. Ultrastruct Pathol 19: 335-345, 1995.

10. Ogawa I, Nikai H, Takata T, et al: Clear cell tumors of minor salivary gland origin. An immunohistochemical and ultrastructural analysis. Oral Surg Oral Med Oral Pathol 72: 200-207, 1991.

11. Yu G, Ma D, Sun K, Li T and Zhang Y: Myoepithelial carcinoma of the salivary glands: behavior and management. Chin Med J (Engl) 116: 163-165, 2003.

12. Zarbo RJ: Salivary gland neoplasia: a review for the practicing pathologist. Mod Pathol 15: 298-323, 2002. 